\title{
LIGATION-BASED MUTATION DETECTION AND RCA IN SURFACE UN-MODIFIED OSTE+ POLYMER MICROFLUIDIC CHAMBERS
}

\author{
Farizah Saharil ${ }^{1}$, Annika Ahlford ${ }^{2}$, Malte Kühnemund ${ }^{2}$, Maciej Skolimowski $^{3}$, Alvaro Conde ${ }^{4}$, \\ Martin Dufva ${ }^{3}$, Mats Nilsson ${ }^{2}$, Monica Brivio ${ }^{2}$, Wouter van der Wijngaart ${ }^{1}$ and Tommy Haraldsson ${ }^{1}$ \\ ${ }^{1}$ KTH Royal Institute of Technology, Dept. of Micro and Nanosystems, SWEDEN \\ ${ }^{2}$ Molecular diagnostics, Science for Life Laboratory, Stockholm University, SWEDEN \\ ${ }^{3}$ DTU Nanotech, Technical University of Denmark, DENMARK \\ ${ }^{4}$ Universidad Nacional de Tucumán, ARGENTINA
}

\begin{abstract}
For the first time, we demonstrate DNA mutation detection in surface un-modified polymeric microfluidic chambers without suffering from bubble trapping or bubble formation. Microfluidic devices were manufactured in off-stoichiometry thiol-ene epoxy (OSTE+) polymer using an uncomplicated and rapid fabrication scheme. The device performance was compared to that of PMMA and PDMS devices. In OSTE + devices, we were able to perform ligation-based mutation detection and rolling circle amplification (RCA) assays directly on the un-modified surface without suffering from bubble formation or enzyme inhibition during bio-operation at elevated temperatures. In contrast, PMMA, PDMS and COP microfluidic devices required specific surface treatment.
\end{abstract}

\section{KEYWORDS}

Molecular diagnostics, rolling circle amplification, RCA, epoxy, microfluidics, lab-on-a-chip, OSTE+, offstoichiometry thiol-ene, polymer

\section{INTRODUCTION}

Labs-on-a-chip for molecular diagnostics have had limited application success beyond research, particularly due to lack of appropriate device materials and the difficulty to establish complex molecular assays in microfluidic chip formats. Molecular assays based on ligation of padlock probes [1] for target recognition and subsequent rolling-circle-amplification (RCA) [2] are well-established assays used for rapid, highly sensitive and specific detection of nucleic acids, proteins and bacteria in molecular diagnostics of cancer and infectious disease. The assay specificity allows for high-resolution sequence detection. The digital nature of the detection of the large amplification products makes the assay a promising candidate for single-molecule detection in cells and in solution [3]. The specific dual recognition of one single padlock probe per target, followed by the independent amplification of each target, enables multiplex assay development with little optimization required as compared to polymerase chain reaction (PCR). Furthermore, the protocols are uncomplicated with successive additions of reagents and the isothermal nature of the RCA reaction abolishing the need for fast temperature cycling. For most mass-market applications, the cost of lab-on-a-chip diagnostic devices needs to be limited, making polymer replication the only potentially commercially viable manufacturing technique.
Recently, we performed highly specific and sensitive exponential circle-to-circle amplification (C2CA, Figure 1) [3-4] in microfluidic chambers manufactured using PDMS casting [5], micromilling of PMMA [6] and injection molding of COP. However, sub-optimal fluidic behaviour, including bubble trapping and bubble formation, was observed in these structures when no surface modification was employed prior to the assay.

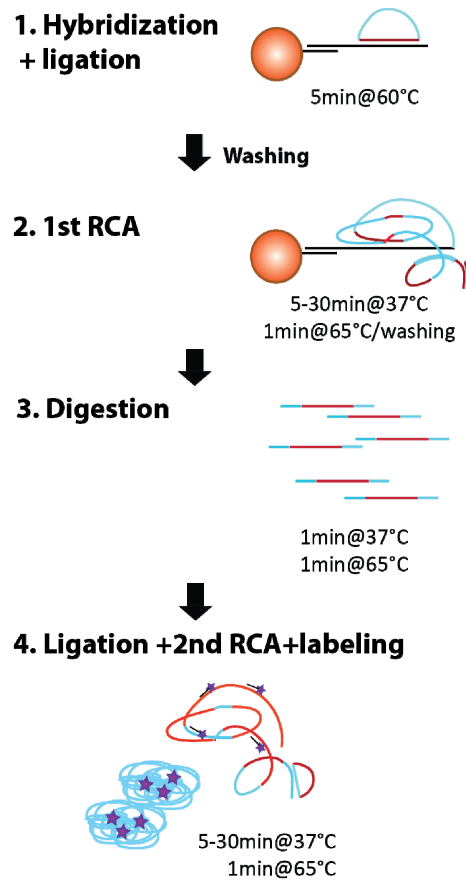

Figure 1: Schematic of the DNA detection assay: (1) The target sequence is captured to a solid support (magnetic beads). Upon recognition of the target sequence the padlock probe (red-blue) is circularized and enzymatically ligated. (2) The template is amplified by RCA using the padlock as template. (3) The long concatenated RCA products are restriction digested. (4) The monomers are re-circularized and used as templates for the second RCA and labelled in real-time by hybridization of fluorescent probes.

The recently introduced OSTE+ dual cure polymer system [7-9] has shown attractive characteristics for microfluidic applications, including a fast turn-around fabrication process, tuneable mechanical and surface properties, and the ability of the material to adhesive-free bond to a large range of substrates, including itself, by adjusting the amount of free thiol and epoxy surface groups in the polymer. 
A

(1)
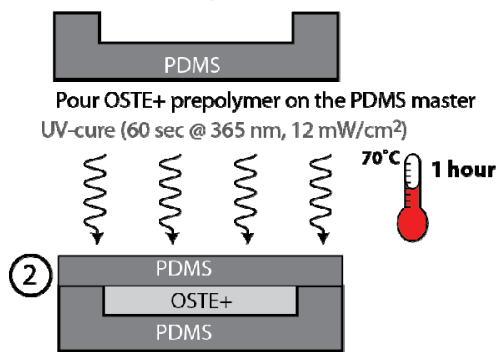

Form a hard top cover by initiating the thiol-ene reaction by UV and the epoxy-thiol by heating to $70^{\circ} \mathrm{C}$ for $1 \mathrm{~h}$.

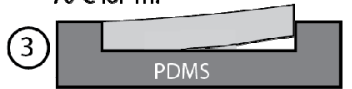

Peel off the hard and fully cured polymer layer which now exposes hydroxyl groups.

(4)

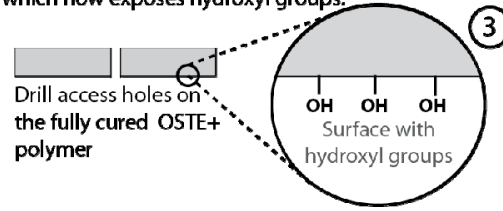

(1)

Bottom polymer layer

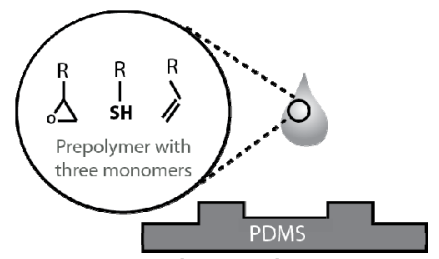

Pour OSTE+ prepolymer on the PDMS master

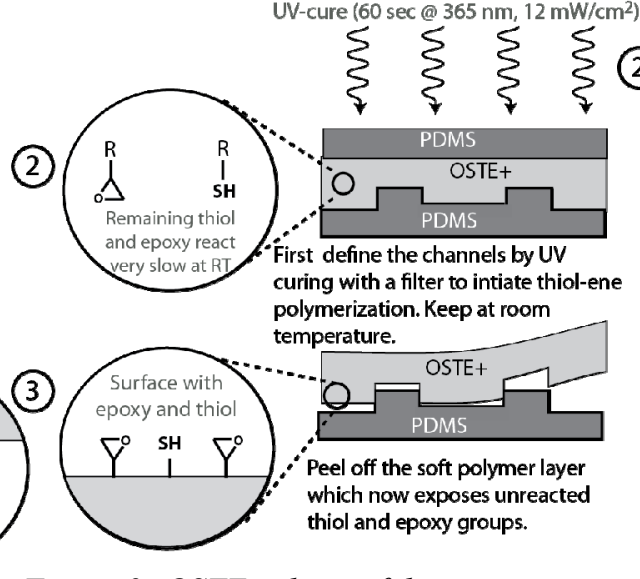

C

(1) Bonding of polymer chip

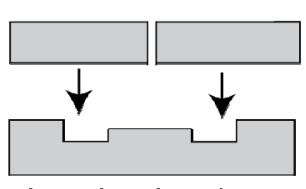

Align and attach to substrate

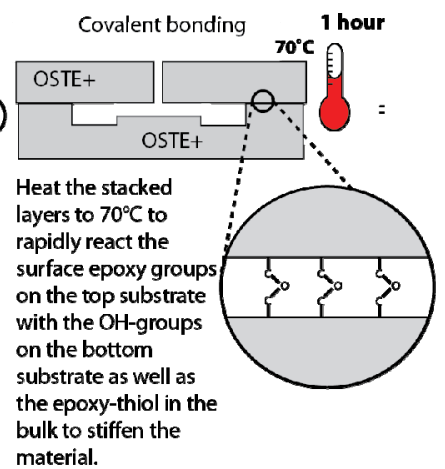

Figure 2: OSTE + device fabrication process

Furthermore, OSTE + has a high glass transition temperature $\left(75^{\circ} \mathrm{C}<\mathrm{T}_{\mathrm{g}}<115^{\circ} \mathrm{C}\right)$ making it suitable as recipient material for molecular assays that require enzyme incubation at elevated temperatures, such as the C2CA protocol. However, OSTE+ has not been tested for its compatibility with DNA amplification processes previously. The focus of this work was therefore to investigate the need for surface functionalization prior to performing RCA bioassays.

\section{FABRICATION}

OSTE + chips of size $76 \times 29 \times 1 \mathrm{~mm}^{3}$ containing multiple individual reaction chambers of volumes ranging between 5-40 $\mu \mathrm{l}$ were fabricated using thiol (Pentaerythritol tetrakis (2-mercaptoacetate)), allyl (triallyl-1,3,5-triazine-2,4,6(1H,3H,5H)-trione) (material provided by Mercene Labs AB, Sweden), epoxy (Bisphenol A diglycidylether (BADGE) from SigmaAldrich, Germany), $0.1 \%$ Lucirin TPO and $0.1 \%$ photo latent based initiator (from BASF, Switzerland) at a stoichiometric ratio of 1/1.8/0.8 . OSTE+ allows for a two-step fabrication process: 1) after casing liquid prepolymer on a mold, a first UV curing step allows for the solidification of the polymer by a rapid thiol-ene reaction and demolding of the polymer layer; 2) a second, thermally accelerated, epoxy-thiol, curing process, enables covalent bonding of the polymer to almost any other substrate material.

Our microfluidic devices consisted of two covalently bonded OSTE+ layers. First, the unstructured top layer is fabricated by pouring a freshly mixed OSTE + prepolymer on a flat PDMS master. Subsequent UV exposure and thermal treatment initiate both curing processes and result in a hard layer. Fluidic access ports are easily drilled through the layer. The fully cured OSTE+ material surface has now hydroxyl groups exposed (Figure 2 A:13 ). The device bottom layer is fabricated by first UV- curing the polymer on a patterned PDMS master. This leaves a soft, non-sticky, solid polymer with unreacted epoxy and thiol groups on its surface and in its bulk (Figure 2 B:1-4). The top and bottom layers are thereafter put into contact and heated in an oven to covalently react the hydroxyl groups with the unreacted epoxy groups (Figure $2 \mathrm{C}$ ). The final chip consists of a stiff thermoset polymer with mechanical properties similar to those of PMMA but with resistance to solvents, acids and bases.

In previous work, PMMA chambers were fabricated by micromilling followed by solvent assistant bonding [6], and PDMS-glass chips were fabricated by casting and thermal curing the PDMS on a milled PMMA mold, followed by clamping the PDMS layer to a microscope glass slide [5].

\section{BIOASSAY}

The full C2CA assay consists of 4 sequential steps:

1.Hybridization and ligation: streptavidine-coated Myone Dynabeads ${ }^{\mathrm{TM}}$ (Life Technologies, USA) are conjugated to a biotinylated capture oligonucleotide in salt buffer in a test tube before they are introduced to the OSTE chambers or to $0.2 \mathrm{ml}$ Eppendorf test tube strips. The beads are kept in place using a permanent magnet. A mix containing ligase, padlock probes and synthetic DNA target are added to the tubes or chambers and incubated to allow target and probe hybridization followed by ligation on the magnetic beads. The unreacted probes are washed away.

2.Next, first RCA mixture is added and incubated for target amplification.

3.Thereafter, an enzymatic treatment by Alu1 (NEB, USA) restriction enzyme is performed to release (digest) the RCA products from the beads.

4.Finally, a second round of RCA and fluorescent labelling are performed.

The assay steps were performed in the OSTE+ 
microfluidic chambers or in $0.2 \mathrm{ml}$ Eppendorf test tube strips. All incubation times and temperatures are stated in Figure 1. Counting of RCA products was performed in a flow channel using a Zeiss LSM 510 META confocal microscope.

We performed three types of experiments. In the first experiment, assay step 1 (hybridization and ligation) is performed in the OSTE+ microfluidic device, and all other steps are performed in test tubes. In the second experiment, assay step 1, 3 and 4 are performed in test tubes, whereas assay step 2 (the first RCA) is performed in the OSTE+ device. In the third experiment, all assay steps are performed in test tubes. For all three experiments, negative control tests were performed.

\section{EVALUATION AND DISCUSSION}

To evaluate the microfluidic devices we first visually checked for bubble formation, for leakage and for structural deformation. Thereafter we checked the bioassay performance in the devices.

In previous work (Figure $3 \mathrm{~A}$ and $3 \mathrm{~B}$ ), gas bubbles were observed when filling untreated PMMA, PDMS and COP microfluidic devices. Even if bubble-free priming could be observed during some careful priming experiments, gas bubbles would occur at elevated temperatures. This is not surprising, as PMMA, PDMS and COP are hydrophobic in their native state. Moreover,
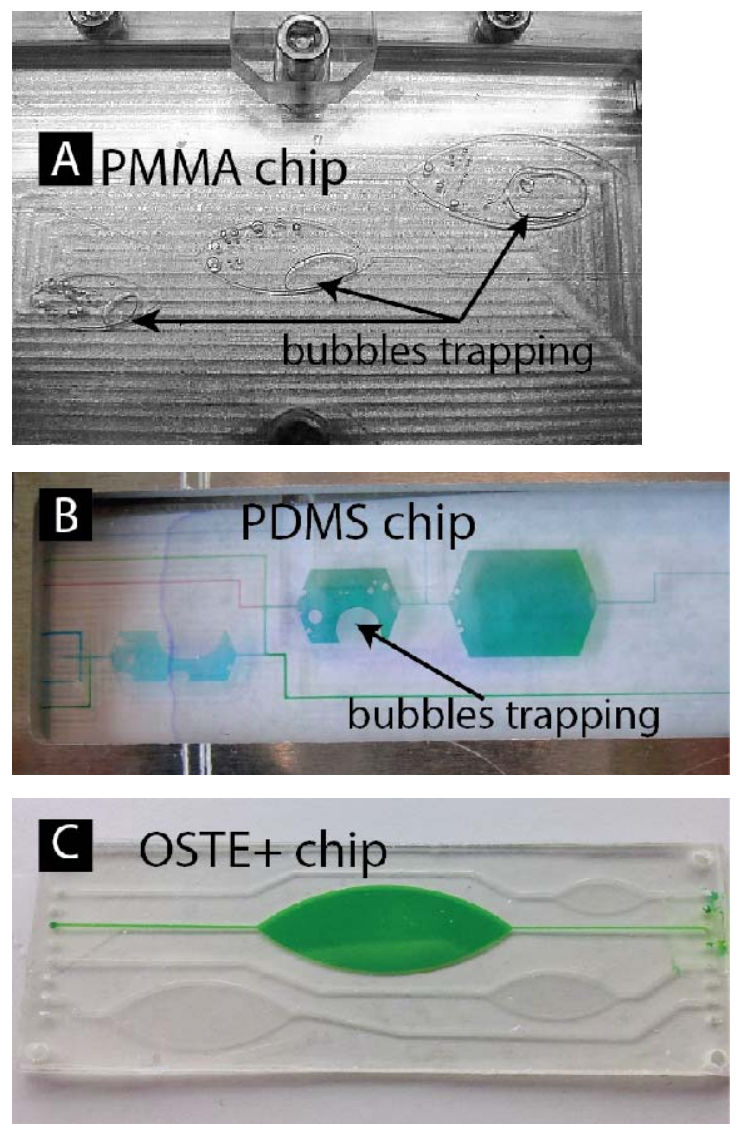

Figure 3: Pictures taken after priming experiments in three polymer chips: (A) Bubble occurence in untreated PMMA microchambers. (B) Bubble occurence in untreated PDMS microchambers (C). OSTE+ microfluidic chips without any surface modification allow for bubble free operation at elevated temperatures.
PDMS is highly permeable for gases, and influx of air will aggravate the bubble formation.

In this work, liquid priming OSTE+ devices was performed by capillary filling with dyed water, after which the device was heated to $60^{\circ} \mathrm{C}$ for $60 \mathrm{~min}$. No bubbles could be detected, as seen in Figure 3C. This desirable fluidic behaviour can be described to the hydrophilicity of the OSTE+, which' surface exhibits a high density of hydroxyl groups formed in the thiol-epoxy reaction. Also, neither structural deformation nor leakage could be observed during or after this experiment. The bioassay performance was evaluated by counting the amount of $\mathrm{C} 2 \mathrm{CA}$ product generated. Figure 4 shows the incubation of reagents during a ligation reaction in the OSTE+ chip.

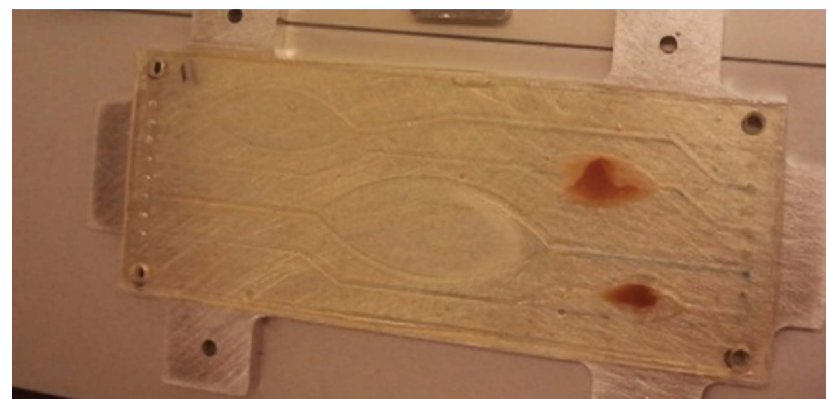

Figure 4: Ligation mix in the OSTE+ chip. The ligation mix is colourless, but the rust-coloured magnetic beads can be easily discerned in the microfluidic chambers.

Successful results were obtained from all three experiments. The amount of $\mathrm{C} 2 \mathrm{CA}$ product generated was similar in all three experiments (Figure 5), indicating that the efficiency of the hybridisation and ligation step and of the RCA step inside the OSTE+ microfluidic chambers was similar as that in the test tubes. Also, the positive / negative control count ratio is $>100$ for all experiments.

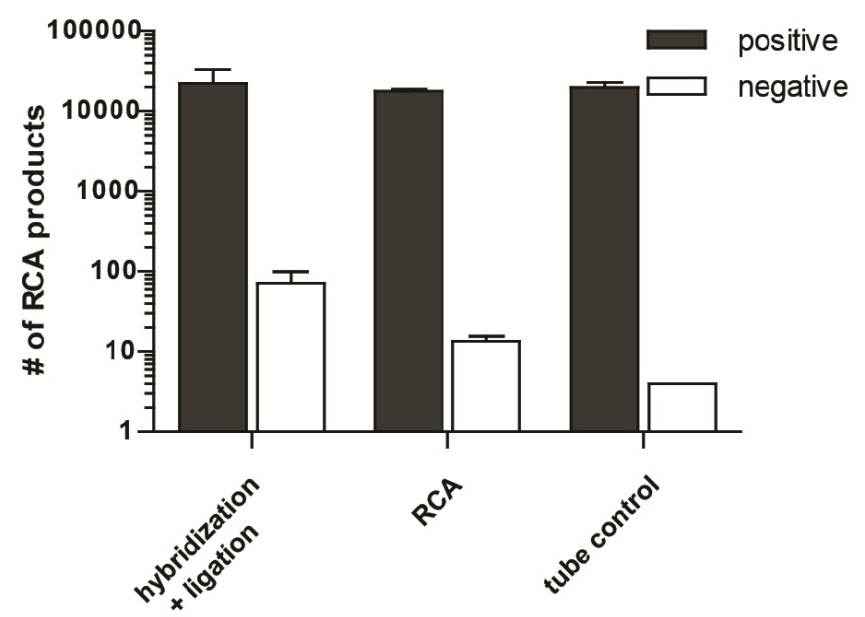

Figure 5: RCA product count the three experiments. The left bars show the result for the first assay experiment, with the hybridization and ligation performed in the OSTE+ device; the middle bars show the result for the second assay experiment, with the first RCA performed in the OSTE + device; the right bars show the result when all assay steps are performed in test tubes. 


\section{CONCLUSIONS}

We have demonstrated that untreated OSTE+ has excellent surface properties for performing nucleic acid detection using RCA, making it a preferable material for manufacturing microfluidic devices for bioassays. Currently we are systematically optimizing each reaction step of the protocol and implementing the full procedure into an integrated microfluidic format.

\section{ACKNOWLEDGEMENTS}

This work was financed in part by the EU sponsored project IMI RAPP-ID.

\section{REFERENCES}

[1] M. Nilsson et al., "Padlock probes reveal singlenucleotide differences, parent of origin and in situ distribution of centromeric sequences in human chromosomes 13 and 21", Nat Genet., vol. 16, pp. 252-255, 1997.

[2] J. Baner et al., "Signal amplification of padlock probes by rolling circle replication", NAR, vol. 26 , pp. 50735078, 1998.

[3] J. Jarvius et al., "Digital quantification using amplified single-molecule detection", Nat Meth, vol. 3, pp. 725727, 2006.

[4] F. Dahl et al., "Circle-to-circle amplification for precise and sensitive DNA analysis", PNAS, vol. 101, pp. 4548-4553, 2004.

[5] A. Ahlford et al., "A microfluidic platform for personalized cancer diagnostics by padlock probe ligation and circle-to-circle amplification", Proc. $\mu$ TAS conf, Oct 2011, pp. 61-63.

[6] D. Sabourin, J. Petersen, D. Snakenborg, M. Brivio, H. Gudnadson, A. Wolff and M. Dufva, "Microfluidic DNA microarrays in PMMA chips: streamlined fabrication via simultaneous DNA immobilization and bonding activation by brief UV exposure", Biomedical Microdevices, vol. 12, pp. 673-681, (2010).

[7] F. Saharil et al., "Dry transfer bonding of porous silicon membranes to OSTE $(+)$ polymer microfluidic devices", Proc. MEMS conf., Jan 2012, pp. 232-234.

[8] F. Saharil et al., "Dry adhesive bonding of nanoporous inorganic membranes to microfluidic deices using the OSTE(+) dual-cure polymer", J. Micromech. Microeng., vol. 23, pp. 025021, 2013.

[9] F. Saharil et al., "Superior dry bonding of OSTE $(+)$ polymers for heterogeneous material labs-on-chip", Proc. $\mu$ Tas conf., Oct 2012, pp. 1831-1833. 